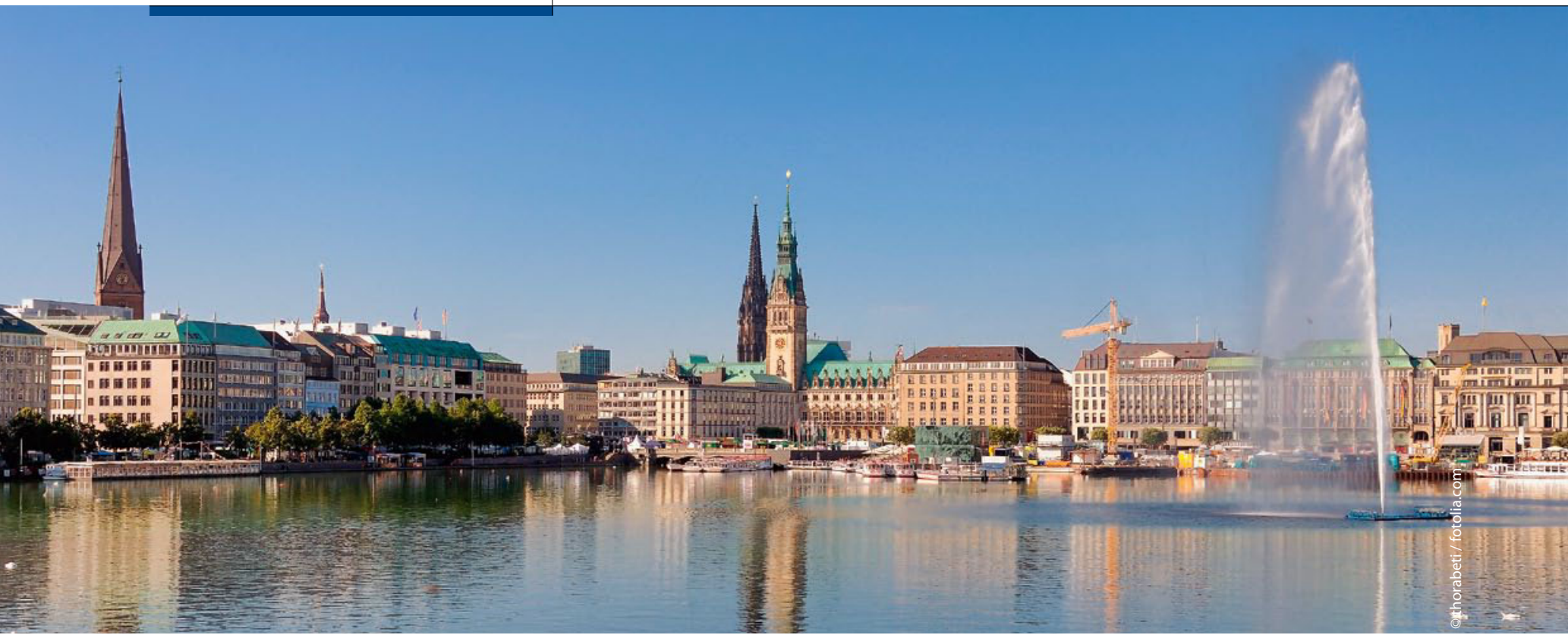
\title{
Die Zukunft der Urologie
}

63. Kongress der Deutschen Gesellschaft für Urologie in Hamburg

\author{
Unter dem Motto „Zukunft sichern - Talente fördern“ lud die Deutsche Gesellschaft für Urologie in \\ diesem Jahr zum 63. DGU-Kongress nach Hamburg ein. Neben aktuellen Forschungsergebnissen \\ rund um urologische Krankheitsbilder wie überaktive Blase und Prostata- oder Nierenkarzinom \\ stand auch die Rekrutierung von qualifiziertem Nachwuchs im Mittelpunkt des Interesses.
}

\section{Nierenteilresektion statt Nephrektomie}

$\mathrm{N}$ icht nur bei früh erkannten Nierentumoren sollte das Organ möglichst erhalten werden. „Auch in Anbetracht der längeren Überlebenszeiten durch die neuen medikamentösen Therapien ist eine Nierenteilresektion mittlerweile eine immer häufiger genutzte Methode“, erläuterte PD Dr. Christian Doehn, Lübeck. Für die organschonende Technik sprechen der Erhalt von funktionstüchtigem Nierenparenchym und somit die Vermeidung hyperfiltrationsbedingter Nierenschäden. Darüber hinaus konnte beobachtet werden, dass die Rate an Patienten mit chronischem Nierenversagen und allen damit verbundenen Auswirkungen auf die Lebensqualität nach radikaler Nephrektomie größer ist als nach einer Nierenteilresektion. Ein weiteres Argument ist die onkologische Sicherheit der Methode.

In den aktuellen EAU-Leitlinien für Nierenzellkarzinome gilt die Nierenteil- resektion bei organbegrenzten Tumoren $<4 \mathrm{~cm}$ und gesunder kontralateraler Niere als Standardtherapie. Diese Technik führt zu vergleichbar guten Ergebnissen wie eine Nephrektomie, das heißt zu tumorspezifischen 5-Jahres-Überlebensraten über $90 \%$. In urologischen Zentren wird sogar eine Teilresektion bei Tumoren $>4 \mathrm{~cm}$ favorisiert, wenn sie günstig lokalisiert sind.

Verschiedene Methoden bieten sich zur Nierenteilresektion an. Aus onkologischer Sicht zeigt sich in Bezug auf das tumorspezifische Überleben vor allem bei kleinen Tumoren bis zu einer Größe von $4 \mathrm{~cm}$ kein Unterschied zwischen der offenen und laparoskopischen Operationstechnik [Lane BR, Gill IS. J Urol 2010; 183: 473-9]. Welche Operationsmethode angewendet wird, hängt von der Indikation, den individuellen Erfordernissen des Patienten und der chirurgischen Er- fahrung des Operateurs ab. Es sollte diejenige Methode eingesetzt werden, die für den Patienten das beste Ergebnis verspricht. Dies bestätigte auch Thomas Jankowski, Homburg, der noch einen Schritt weiter ging und die offene und die Roboterassistierte Nierenteilresektion miteinander verglich. Bei sorgfältiger Indikationsstellung konnten bei den 50 ersten Nierenteilresektionen durch das daVinci-System adäquate onkologische und funktionelle Ergebnisse im Vergleich zur offenen Operation erzielt werden. Allerdings warnte der Urologe abschließend: „Wichtig ist es, nicht die Lernkurve am Roboter zu unterschätzen - besonders wenn zufriedenstellende Ischämiezeiten als oberste Priorität angesehen werden“"

Bettina Reich

P 10: „Nieren- und NebennierentumorenTherapie" 